\title{
THE BOUNDEDLY CONTROLLED WHITEHEAD THEOREM
}

\author{
DOUGLAS R. ANDERSON AND HANS JØRGEN MUNKHOLM
}

(Communicated by James E. West)

\begin{abstract}
This note contains a version of the Whitehead Theorem for boundedly controlled maps of $\mathrm{CW}$ complexes that is often useful in applications and complements the Whitehead Theorem in our book Boundedly controlled topology (Lecture Notes in Math., vol. 1323, Springer-Verlag, 1988). We also include a version of the Whitehead Theorem valid for simply connected boundedly controlled $\mathrm{CW}$ complexes.
\end{abstract}

\section{INTRODUCTION}

In our book [AM1] we state and prove a version of the Whitehead Theorem that allows one to decide whether a map $f:(X, p) \rightarrow(Y, q)$ between boundedly controlled (bc) $\mathrm{CW}$ complexes is a bc homotopy equivalence. The version of the Whitehead Theorem given there [p. 93 $]^{1}$ is phrased entirely in terms of homotopy. In applications it is often useful to have a version that is phrased in terms of conditions on the low dimensional homotopy and on the homology of the universal cover. For example, this is the version needed by Vogell in [V]. We prove such a Whitehead Theorem in this paper. The version of the Whitehead Theorem proved in [AM1] is the one involving conditions (1), (2), and (3) in the following theorem.

Boundedly Controlled Whitehead Theorem. Let $f:(X, p) \rightarrow(Y, q)$ be a bc map between finite-dimensional bc $C W$ complexes over the boundedness control space $Z$. Then $f$ is a bc homotopy equivalence if and only if

(1) $(Y, q)$ is coextensive with $(X, p)$;

(2) $f_{*}: \pi_{n}^{c}(X, p) \rightarrow f^{!} \pi_{n}^{c}(Y, q)$ is an isomorphism for $n=0$ and 1; and either

(3) $f_{*}: \pi_{n}^{c}(X, p) \rightarrow f^{!} \pi_{n}^{c}(Y, q)$ is an isomorphism for $n \geq 2$; or

(3') $\widetilde{f}_{*}: H_{n}^{F}(\widetilde{X}) \rightarrow f^{!} H_{n}^{F}(\widetilde{Y})$ is an isomorphism for all $n \geq 0$.

In this theorem $\tilde{X}$ and $\tilde{Y}$ are not the usual universal covers of $(X, p)$ and $(Y, q)$ respectively, but are "fragmented versions" of them. These are described

Received by the editors March 2, 1990 and, in revised form, June 10, 1991.

1980 Mathematics Subject Classification (1985 Revision). Primary 55P10.

The first author was partially supported by the NSF under grant number DMS-8803149.

The second author was partially supported by SNF (Denmark) under grant number 11-7792.

${ }^{1}$ References to the book [AM1] are given just by page numbers. 
in $\S 1$ (cf. Example 1.12) where we review the ideas from bc topology needed to understand the statement and proof of this theorem. In particular, Lemmas 1.7 and 1.10 give criteria for recognizing when $f_{*}$ and $\tilde{f}_{*}$ in $(2),(3)$, and $\left(3^{\prime}\right)$ are isomorphisms. The proof of the Boundedly Controlled Whitehead Theorem is given in $\S 2$.

A bc CW complex $(X, p)$ is simply connected if $\pi_{n}^{c}(X, p)=0$ for $n=0,1$. If $(X, p)$ is simply connected, its fragmented universal cover is isomorphic to the fragmented $\mathrm{CW}$ complex associated to $(X, p)$ (cf. Example 1.6). In this case, the Boundedly Controlled Whitehead Theorem reduces to the following theorem.

Simply Connected BC Whitehead Theorem. Let $f:(X, p) \rightarrow(Y, q)$ be a $b c$ map between simply connected, finite-dimensional bc $C W$ complexes. Then $f$ is $a$ bc homotopy equivalence if and only if $(Y, q)$ is coextensive with $(X, p)$ and $f_{*}: H_{n}^{c}(X, p) \rightarrow H_{n}^{c}(Y, q)$ is an isomorphism for all $n \geq 0$.

Since $H_{n}^{c}(X, p)$ is just the fragmented homology of the fragmented complex over $\mathfrak{P}$ associated with $(X, p)$, the reader may still use Lemma 1.7 to decide when $f_{*}$ is an isomorphism.

\section{A REVIEW OF BOUNDEDLY CONTROLLED TOPOLOGY}

This section reviews the concepts from [AM1] needed to understand the statement and proof of the Boundedly Controlled Whitehead Theorem.

Let $Z$ be a topological space. $A$ boundedness control structure on $Z$ is a pair $(\mathfrak{P}, C)$ where $\mathfrak{P}$ is a directed family of nonempty subsets of $Z$ ordered by inclusion and $C: \mathfrak{P} \rightarrow \mathfrak{P}$ is an order-preserving function that satisfies certain properties [pp. 41, 42]. Among these are that for every $K \in \mathfrak{P}, K \subseteq C K$, that $Z=\bigcup\left\{C^{n} K \mid n=0,1,2, \ldots\right\}$ and that for every $K \in \mathfrak{P}$, there is a minimal element $K_{0} \in \mathfrak{P}$ with $K_{0} \subseteq K$. A boundedness control space is a space $Z$ together with a boundedness control structure $(\mathfrak{P}, C)$. We denote a boundedness control space simply by $Z$.

Example 1.1. We recall that a metric $\rho: Z \times Z \rightarrow \mathbb{R}_{+}$is proper if $\rho(z,-): Z \rightarrow$ $\mathbb{R}_{+}$is proper for every $z \in Z$. Let $(Z, \rho)$ be a proper metric space for which $B(z, r) \subseteq B(y, s)$ implies that $B(z, r+1) \subseteq B(y, s+1)$. Let $\mathfrak{P}=$ $\{B(z, n) \mid(z, n) \in Z \times \mathbb{N}\}$, and set $C(B(z, n))=B(z, n+1)$. Then $(\mathfrak{P}, C)$ is a boundedness control structure on $Z$ called the metric boundedness control structure.

Let $Z$ be a boundedness control space. A boundedly controlled (or simply, $b c) C W$ complex over $Z$ is a pair $(X, p)$ where $X$ is a $C W$ complex and $p: X \rightarrow Z$ is a continuous map. It is also required that there be an integer $n \geq 0$ so that for every cell $e \in X$ there is a minimal element $K_{e} \in \mathfrak{P}$ with $p(e) \subseteq C^{n}\left(K_{e}\right)$. If $(X, p)$ is a bc CW complex over $Z$ and $K \in \mathfrak{P}$, let $X_{K}$ be the smallest subcomplex of $X$ containing $p^{-1}(K)$.

Let $(X, p)$ and $(Y, q)$ be bc CW complexes over $Z$. We say that $(X, p)$ and $(Y, q)$ are coextensive if there is an integer $m \geq 0$ so that for all $K \in \mathfrak{P}$, if $X_{K} \neq \varnothing$, then $Y_{C^{m} K} \neq \varnothing$ and if $Y_{K} \neq \varnothing$, then $X_{C^{m} K} \neq \varnothing$.

A boundedly controlled (or simply, bc) map $f:(X, p) \rightarrow(Y, q)$ of delay d is a continuous map $f: X \rightarrow Y$ for which there is an integer $d \geq 0$ so that for every $K \in \mathfrak{P}, f\left(X_{K}\right) \subseteq Y_{C^{d} K}$. Let $(X, p) \times I=(X \times I, p \pi)$ where $\pi$ is 
projection on the first factor. Then there are obvious notions of a bc homotopy between bc maps and of $f$ being a bc homotopy equivalence.

Some background is needed to define the algebraic invariants, boundedly controlled homotopy, and fragmented homology that appear in the BC Whitehead Theorem.

A category with endomorphism [p. 3] is a triple $(\mathfrak{B}, C, \tau)$ consisting of a category $\mathfrak{B}$, a functor $C: \mathfrak{B} \rightarrow \mathfrak{B}$, and a natural transformation $\tau: I \rightarrow C$ satisfying $\tau_{C(B)}=C \tau_{B}$ for all objects $B$ of $\mathfrak{B}$. Here $I$ is the identity functor of $\mathfrak{B}$. When $C$ and $\tau$ are clear from the context, we denote a category with endomorphism simply by $\mathfrak{B}$.

Example 1.2. Let $(\mathfrak{P}, C)$ be a boundedness control structure on $Z$, and regard the partially ordered set $\mathfrak{P}$ as a category with morphisms the inclusions. Let $\tau: I \rightarrow C$ be the natural transformation with $\tau(K)$ the inclusion $K \subseteq C K$ for every $K$. Then $(\mathfrak{P}, C, \tau)$ is a category with endomorphism.

The property of being a category with endomorphism reproduces itself under certain constructions.

Example 1.3. Let $G: \mathfrak{B} \rightarrow \mathfrak{G}$ be a functor where $\mathfrak{B}$ is a category with endomorphism and $\mathfrak{G}$ is the category of small groupoids. Let $\mathfrak{B} G$ be the category with objects pairs $(x, B)$ where $x \in G(B)$ and $B \in \mathfrak{B}$ and with morphisms pairs $(\omega, i):(x, B) \rightarrow(y, A)$ where $i: B \rightarrow A$ is a morphism in $\mathfrak{B}$ and $\omega: G(i)(x) \rightarrow y$ is a morphism in $G(A)$. Let $\bar{C}: \mathfrak{B} G \rightarrow \mathfrak{B} G$ be given on objects by $\bar{C}(x, B)=\left(G\left(\tau_{B}\right)(x), C B\right)$ and on morphisms by $\bar{C}(\omega, i)=\left(G\left(\tau_{A}\right)(\omega), C(i)\right)$. Let $\bar{\tau}: I \rightarrow \bar{C}$ be given by $\bar{\tau}(x, B)=\left(1_{x}, \tau_{B}\right)$. Here $I$ is the identity functor. Then $(\mathfrak{B} G, \bar{C}, \bar{\tau})$ is again a category with endomorphism. Notice there is a forgetful functor $\rho: \mathfrak{B} G \rightarrow \mathfrak{B}$ that sends $(x, B)$ to $B$.

Example 1.4. Let $\mathfrak{B}$ be a category with endomorphism, and consider the functor category $\mathfrak{C}^{\mathfrak{B}}$. Then $C$ and $\tau$, respectively, induce $\bar{C}: \mathfrak{C}^{\mathfrak{B}} \rightarrow \mathfrak{C}^{\mathfrak{B}}$ and $\bar{\tau}: I \rightarrow \bar{C}$ by setting $\bar{C}(F)=F C, \bar{C}(\nu)=F\left(\nu_{C}\right)$ when $\nu: F \rightarrow G$ and $\bar{\tau}_{F}=F(\tau)$, respectively. Then $\left(\mathfrak{C}^{\mathfrak{B}}, \bar{C}, \bar{\tau}\right)$ is again a category with endomorphism.

To simplify notation, we usually denote $\bar{C}$ and $\bar{\tau}$ of Examples 1.3 and 1.4 by just $C$ and $\tau$, respectively.

Let $(\mathfrak{B}, C, \tau)$ be a category with endomorphism. For any object $B \in \mathfrak{B}$, set $\tau^{0}(B)=1$ and $\tau^{n}(B)=\tau_{C^{n-1} B} \cdots \tau_{C B} \tau_{B}$ for $n \geq 1$. The collection of morphisms $\Sigma=\left\{\tau^{n}(B) \mid B \in \mathfrak{B}, n \geq 0\right\}$ admits a calculus of left fractions [p. 5], and we may form the category of fractions $\mathfrak{B}\left(\Sigma^{-1}\right)$. Let $Q: \mathfrak{B} \rightarrow \mathfrak{B}\left(\Sigma^{-1}\right)$ be the natural functor. For $B_{i} \in \mathfrak{B}(i=1,2)$, every morphism $h: Q\left(B_{1}\right) \rightarrow$ $Q\left(B_{2}\right)$ has the form $Q\left(\tau^{d}\left(B_{2}\right)\right)^{-1} Q(f)$ for some $f: B_{1} \rightarrow C^{d} B_{2}$. We say that $B \in \mathfrak{B}$ represents $Q(B)$ and that $f$ is a morphism of delay $d$ representing $h$. Thus if $B_{i} \in \mathfrak{B}(i=1,2)$, then their images in $\mathfrak{B}\left(\Sigma^{-1}\right)$ are isomorphic if and only if there are morphisms $f: B_{1} \rightarrow C^{m} B_{2}$ and $g: B_{2} \rightarrow C^{n} B_{1}$ in $\mathfrak{B}$ for which $C^{m}(g) f=\tau^{m+n}=C^{n}(f) g$.

Proposition 1.5. If the category $\mathfrak{B}$ is abelian and $C$ preserves finite products and kernels, then $\mathfrak{B}\left(\Sigma^{-1}\right)$ is abelian. In particular, if $\mathfrak{C}$ is abelian, then $\mathfrak{C}^{\mathfrak{B}}\left(\Sigma^{-1}\right)$ is abelian. 
Proof. The first sentence is Corollary 2.5 [p. 9]. If $\mathfrak{C}$, is abelian, so is $\mathfrak{C}^{\mathfrak{B}}$, and the second sentence follows from the first and Example 1.4.

We are now ready to define fragmented spaces and their homology.

Let $\mathfrak{B}$ be a category with endomorphism and $\mathfrak{C W}$ be the category of $\mathrm{CW}$ complexes. Then $\mathfrak{C W}^{\mathfrak{B}}\left(\Sigma^{-1}\right)$ is called the category of fragmented $C W$ complexes over $\mathfrak{B}$. An object in this category is called a fragmented $C W$ complex over $\mathfrak{B}$ and is represented by a functor $X: \mathfrak{B} \rightarrow \mathfrak{C W}$. We often write $X_{B}$ instead of $X(B)$. If $X, Y: \mathfrak{B} \rightarrow \mathfrak{C W}$ represent fragmented $\mathrm{CW}$ complexes over $\mathfrak{B}$, we say $f: X \rightarrow Y$ is a morphism of delay $d$ if $f$ is represented by a natural transformation $f: X \rightarrow \bar{C}^{d} Y$; i.e., a family of maps $\left\{f_{B}: X_{B} \rightarrow Y_{C^{d} B} \mid B \in \mathfrak{B}\right\}$.

Example 1.6. Let $(X, p)$ be a bc $\mathrm{CW}$ complex over the boundedness control space $(Z, \mathfrak{P}, C)$, and for $K \in \mathfrak{P}$, let $X_{K}$ be the smallest subcomplex of $X$ containing $p^{-1}(K)$. The functor $X^{\prime}: \mathfrak{P} \rightarrow \mathfrak{C W}$ sending $K$ to $X_{K}$ is called the fragmented $C W$ complex over $\mathfrak{P}$ associated with $(X, p)$. If $f:(X, p) \rightarrow(Y, q)$ is a bc map of delay $d$, then the collection of maps $\left\{f\left|X_{K}: X_{K} \rightarrow Y_{C^{d} K}\right| K \in\right.$ $\mathfrak{P}\}$ gives a natural transformation $f^{\prime}: X^{\prime} \rightarrow \bar{C}^{d} Y^{\prime}$ of delay $d$ representing a morphism $X^{\prime} \rightarrow Y^{\prime}$ in $\mathfrak{C W}^{\mathfrak{P}}\left(\Sigma^{-1}\right)$.

Let $X: \mathfrak{B} \rightarrow \mathfrak{C W}$ represent a fragmented $\mathrm{CW}$ complex over $\mathfrak{B}$. For any $n \geq$ 0 , the composite functor $\mathfrak{B} \stackrel{X}{\longrightarrow} \mathfrak{C W} \stackrel{H_{n}}{\longrightarrow} \mathfrak{A b}$ represents an object of $\mathfrak{A b}^{\mathfrak{B}}\left(\Sigma^{-1}\right)$ called the $n$th fragmented homology of $X$ and denoted by $H_{n}^{F}(X)$. Here $\mathfrak{A} \mathfrak{b}$ is the category of abelian groups and $H_{n}$ is the $n$th singular homology group. If $f: X \rightarrow Y$ is a map of fragmented spaces with delay $d$, then the collection $\left\{f_{B^{*}}: H_{n}\left(X_{B}\right) \rightarrow H_{n}\left(Y_{C^{d} B}\right) \mid B \in \mathfrak{B}\right\}$ constitutes a morphism in $\mathfrak{A b}^{\mathfrak{B}}$ representing a morphism $f_{*}: H_{n}^{F}(X) \rightarrow H_{n}^{F}(Y)$.

The proofs of Lemmas 1.7 and 1.10 are contained in the discussion on [pp. 23-29].

Lemma 1.7. Let $f: X \rightarrow Y$ be a morphism of delay $d$ of fragmented spaces over $\mathfrak{B}$. Then $f_{*}: H_{n}^{F}(X) \rightarrow H_{n}^{F}(Y)$ is an isomorphism if and only if there is an integer $m=m(n)$ so that in the diagram

$$
\begin{array}{ccc}
H_{n}\left(X_{B}\right) \stackrel{f(B)_{*}}{\longrightarrow} & H_{n}\left(Y_{C^{d} B}\right) \\
X\left(\tau^{m}(B)\right)_{*} \downarrow & \downarrow X\left(\tau^{m}\left(C^{d}(B)\right)_{*}\right. \\
H_{n}\left(X_{C^{m} B}\right) \stackrel{ }{\underset{f\left(C^{m} B\right)_{*}}{\longrightarrow}} H_{n}\left(Y_{C^{m+d} B}\right)
\end{array}
$$

$\operatorname{ker} f(B)_{*} \subseteq \operatorname{ker} X\left(\tau^{m}(B)\right)_{*}$ and $\operatorname{im} X\left(\tau^{m}\left(C^{d}(B)\right)_{*} \subseteq \operatorname{im} f\left(C^{m} B\right)_{*}\right.$.

The relative homology of a fragmented pair is defined similarly. In this case, $(X, Y)$ is a functor $\mathfrak{B} \rightarrow \mathfrak{C W}^{2}$ into the category of pairs of $\mathrm{CW}$ complexes and $H_{n}^{F}(X, Y)$ is represented by the composite functor $\mathfrak{B} \stackrel{(X, Y)}{\longrightarrow} \mathfrak{C W}^{2} \stackrel{H_{n}}{\longrightarrow} \mathfrak{A} \mathfrak{b}$. Furthermore, the family of homomorphisms $\left\{\partial: H_{n}\left(X_{K}, Y_{K}\right) \rightarrow H_{n-1}\left(Y_{K}\right)\right\}$ represents a morphism $\partial: H_{n}^{F}(X, Y) \rightarrow H_{n-1}^{F}(Y)$ that fits into a long exact sequence

$$
\begin{gathered}
\cdots \longrightarrow H_{n+1}^{F}(X, Y) \stackrel{\partial}{\longrightarrow} H_{n}^{F}(Y) \stackrel{i_{*}}{\longrightarrow} H_{n}^{F}(X) \\
\stackrel{j_{*}}{\longrightarrow} H_{n}^{F}(X, Y) \stackrel{\partial}{\longrightarrow} H_{n-1}^{F}(Y) \longrightarrow \cdots
\end{gathered}
$$


where $i_{*}$ and $j_{*}$ are induced by the inclusions.

Example 1.9. Let $X: \mathfrak{B} \rightarrow \mathfrak{C W}$ represent a fragmented $\mathrm{CW}$ complex, $G: \mathfrak{C W} \rightarrow$ $\mathfrak{G}$ assign to each CW complex its fundamental groupoid, and $\mathfrak{B} G(X)$ be the category of Example 1.3. This category is called the fundamental groupoid of $X$ (with some abuse of language). Its objects are pairs $(x, B)$ where $x \in X_{B}$ and $B \in \mathfrak{B}$, and its morphisms are pairs $(\omega, i):(x, B) \rightarrow(y, A)$ where $i: B \rightarrow A$ is a morphism in $\mathfrak{B}$ and $\omega$ is a homotopy class of paths from $y$ to $X(i)(x)$ in $X_{A}$ where the homotopy is modulo endpoints. Notice that in $\mathfrak{B} G(X)$, $\pi_{1}\left(X_{B}, x\right)$ is the group of self-maps of $(x, B)$.

Let $X: \mathfrak{B} \rightarrow \mathfrak{C W}$ be a fragmented $\mathrm{CW}$ complex and $\mathfrak{B} G(X)$ be its fundamental groupoid. Then $\pi_{n}^{F}(X)$ is the object of $\mathfrak{C}^{\mathfrak{B} G(X)}\left(\Sigma^{-1}\right)$ represented by the functor $\pi_{n}: \mathfrak{B} G(X) \rightarrow \mathfrak{C}$ that sends $(x, B)$ to $\pi_{n}\left(X_{B}, x\right)$, the $n$th homotopy group (or pointed set, if $n=0)$ of $\left(X_{B}, x\right)$. Here $\mathfrak{C}$ is the category of pointed sets (if $n=0$ ), groups (if $n=1$ ), or abelian groups (if $n \geq 2$ ). $\pi_{n}^{F}(X, p)$ is called the $n$th fragmented homotopy of $X$. Since $\pi_{1}\left(X_{B}, x\right)$ is the group of self-maps of $(x, B)$ in $\mathfrak{B} G(X), \pi_{n}^{F}(X)$ has the actions of all these "local" fundamental groups built into it.

Let $f: X \rightarrow Y$ be a morphism of delay $d, f_{\sharp}: \mathfrak{B} G(X) \rightarrow \mathfrak{B} G(Y)$ be the functor sending $(x, B)$ to $\left(f_{B}(x), C^{d} B\right)$ and $f^{!}: \mathfrak{C}^{\mathfrak{B} G(Y)}\left(\Sigma^{-1}\right) \rightarrow \mathfrak{C}^{\mathfrak{B} G(X)}\left(\Sigma^{-1}\right)$ be induced by precomposition with $f_{\sharp}$. The family $\left\{f_{*}: \pi_{n}\left(X_{B}, x\right) \rightarrow\right.$ $\left.\pi_{n}\left(Y_{C^{d} B}, f(x)\right) \mid(x, B) \in \mathfrak{B} G(X)\right\}$ represents a morphism

$$
f_{*}: \pi_{n}^{F}(X) \rightarrow f^{!} \pi_{n}^{F}(Y)
$$

in $\mathfrak{C}^{\mathfrak{B} G(X)}\left(\Sigma^{-1}\right)$. It follows from [p. 61] that $f^{4}$ and $f_{*}$ are independent of $d$ in the sense that they are unique up to a canonical equivalence.

If $X^{\prime}$ is the fragmented CW complex over $\mathfrak{P}$ associated with the bc $\mathrm{CW}$ complex $(X, p)$ over $Z$ as in Example 1.6, we call $\mathfrak{P} G\left(X^{\prime}\right)$ the fundamental groupoid of $(X, p)$ and denote this category by $\mathfrak{P} G(X, p)$. Similarly, we denote $\pi_{n}^{F}\left(X^{\prime}\right)$ by $\pi_{n}^{c}(X, p)$ and call this the $n$th $b c$ homotopy of $(X, p)$. It is represented by the functor that sends $(x, K) \in \mathfrak{P} G(X, p)$ to $\pi_{n}\left(X_{K}, x\right)$. If $f:(X, p) \rightarrow(Y, q)$ is a bc map of delay $d$, then the family of homomorphisms $\left\{f_{*}: \pi_{n}\left(X_{K}, x\right) \rightarrow \pi_{n}\left(Y_{C^{d} K}, f(x)\right) \mid(x, B) \in \mathfrak{P} G(X)\right\}$ represents

$$
f_{*}: \pi_{n}^{c}(X, p) \rightarrow f^{!} \pi_{n}^{c}(Y, q) .
$$

Lemma 1.10. Let $f:(X, p) \rightarrow(Y, q)$ be a bc map of delay $d$ between $b c$ $C W$ complexes over the boundedness control space $Z$. Then $f_{*}: \pi_{n}^{c}(X, p) \rightarrow$ $f^{\prime} \pi_{n}^{c}(Y, q)$ is an isomorphism if and only if there is an integer $m=m(n)$ so that in the diagram

$$
\begin{array}{ccc}
\pi_{n}\left(X_{K}, x\right) & f_{0 *} & \pi_{n}\left(Y_{C^{d} K}, f(x)\right) \\
j_{0 *} \downarrow & \downarrow^{j_{1 *}} \\
\pi_{n}\left(X_{C^{m} K}, x\right) & \underset{f_{1 *}}{\longrightarrow} \pi_{n}\left(Y_{C^{m+d} K}, f(x)\right)
\end{array}
$$

$\operatorname{ker} f_{0 *} \subseteq \operatorname{ker} j_{0 *}$ and $\operatorname{im} j_{1 *} \subseteq \operatorname{im} f_{1_{*}}$. (If $n=0$, the first condition is easily seen to imply that if $\xi, \zeta \in \pi_{n}\left(X_{K}, x\right)$ have $f_{0 *}(\xi)=f_{0 *}(\zeta)$, then $j_{0 *}(\xi)=j_{0 *}(\zeta)$.)

Here $j_{0}:\left(X_{K}, x\right) \rightarrow\left(X_{C^{m} K}, x\right)$ and $j_{1}:\left(Y_{C^{d} K}, f(x)\right) \rightarrow\left(Y_{C^{d+m} K}, f(x)\right)$ are the inclusions. 
The reader should be able to formulate the analogous lemma describing when a map of fragmented spaces induces an isomorphism $f_{*}: \pi_{n}^{F}(X) \rightarrow f^{\prime} \pi_{n}^{F}(Y)$.

Let $(Y, X): \mathfrak{B} \rightarrow \mathfrak{C W}^{2}$ be a pair of fragmented $\mathrm{CW}$ complexes over $\mathfrak{B}$. Then $\pi_{n}^{F}(Y, X)$ is the object of $\mathfrak{C}^{\mathfrak{B} G(X)}\left(\Sigma^{-1}\right)$ represented by the functor $\pi_{n}(Y, X): \mathfrak{B} G(X) \rightarrow \mathfrak{C}$ that sends $(x, B)$ to $\pi_{n}\left(Y_{B}, X_{B}, x\right)$. Here $\mathfrak{C}$ is the category of pointed sets (if $i=0,1$ ), groups (if $i=2$ ), or abelian groups (if $i \geq 3)$. The family $\left\{\partial: \pi_{n}\left(Y_{B}, X_{B}, y\right) \rightarrow \pi_{n-1}\left(X_{B}, y\right)\right\}$ represents a morphism $\partial: \pi_{n}^{F}(Y, X) \rightarrow \pi_{n-1}^{F}(X)$ that fits into a long exact sequence

$$
\begin{aligned}
\cdots & \longrightarrow \pi_{n+1}^{F}(Y, X) \stackrel{\partial}{\longrightarrow} \pi_{n}^{F}(X) \stackrel{i_{*}}{\longrightarrow} i^{\prime} \pi_{n}^{F}(Y) \\
& \stackrel{j_{*}}{\longrightarrow} \pi_{n}^{F}(Y, X) \stackrel{\partial}{\longrightarrow} \pi_{n-1}^{F}(X) \longrightarrow \cdots
\end{aligned}
$$

Example 1.12. Let $X: \mathfrak{B} \rightarrow \mathfrak{C W}$ represent a fragmented complex, and let $\mathfrak{B} G(X)$ be its fundamental groupoid as in Example 1.9. If $(x, B) \in \mathfrak{B} G(X)$, let $p(x, B):(\tilde{X}(x, B), \tilde{x}) \rightarrow(X(B), x)$ be the terminal point map where $(\widetilde{X}(x, B), \tilde{x})$ is $\left(P\left(X_{B}, x\right) / \simeq, \varepsilon_{x}\right), P\left(X_{B}, x\right)$ be the space of paths in $X_{B}$ that begin at $x, \simeq$ be the relation of homotopy modulo endpoints, and $\varepsilon_{x}$ is the constant path at $x$. We note that for every $(x, B), p(x, B): \widetilde{X}(x, B) \rightarrow X_{B}$ is the universal cover of the component of $X_{B}$ that contains $x$. If $(\omega, i):(x, B)$ $\rightarrow(y, A)$ in $\mathfrak{B} G(X)$, let $\widetilde{X}(\omega, i): \widetilde{X}(x, B) \rightarrow \widetilde{X}(y, A)$ be the map that sends $\alpha$ to $\omega X(i)(\alpha)$. Then $\widetilde{X}: \mathfrak{B} G(X) \rightarrow \mathfrak{C W}$ represents an object of $\mathfrak{C W}^{\mathfrak{B} G(X)}\left(\Sigma^{-1}\right)$ called the fragmented universal cover of $X$ and denoted also by $\widetilde{X}$. Clearly, $\widetilde{X}$ is simply connected in the sense that $\pi_{n}^{F}(\widetilde{X})=0$ for $n=0,1$.

\section{Proof of the Boundedly Controlled Whitehead Theorem}

The proof of the Boundedly Controlled Whitehead Theorem requires two lemmas. Let $\mathfrak{B}$ be a category with endomorphism and $(Y, X): \mathfrak{B} \rightarrow \mathfrak{C W}$ be a pair of fragmented $\mathrm{CW}$ complexes over $\mathfrak{B}$. Let $\widetilde{Y}: \mathfrak{B} G(Y) \rightarrow \mathfrak{C W}$ be the universal cover of $Y$, and for each $(x, K)$ with $x \in X_{K}$, let $\bar{X}(x, K)=$ $p_{(x, K)}^{-1}\left(X_{K}\right)$ where $p_{(x, K)}:(\tilde{Y}(x, K), \tilde{x}) \rightarrow\left(Y_{K}, x\right)$ is the map of Example 1.12. Then $(x, K) \mapsto(\tilde{Y}(x, K), \bar{X}(x, K))$ is a pair of fragmented $\mathrm{CW}$ complexes over $\mathfrak{B}^{\prime}=\mathfrak{B} G(X)$, which we denote by $\left(f^{\prime} \widetilde{Y}, \bar{X}\right)$ since the functor $(x, K) \mapsto$ $(\widetilde{Y}(x, K), \tilde{x})$ is just $f^{!} \widetilde{Y}$. Let $\mathfrak{B}^{\prime \prime}=\mathfrak{B}^{\prime} G(\bar{X})$ and $\rho: \mathfrak{B}^{\prime \prime} \rightarrow \mathfrak{B}^{\prime}$ be the forgetful functor of Example 1.3.

Lemma 2.1. Let $(Y, X)$ be a pair of fragmented $C W$ complexes over $\mathfrak{B}$ and $f$ : $X \rightarrow Y$ be the inclusion, and suppose

(a) $Y$ is coextensive with $X$ and

(b) $f_{*}: \pi_{n}^{F}(X) \rightarrow f^{!} \pi_{n}^{F}(Y)$ is an isomorphism for $n=0$ and 1 .

Then $\rho_{!} \pi_{n}^{F}\left(f^{!} \tilde{Y}, \bar{X}\right)=\pi_{n}^{F}(Y, X)$ for all $n \geq 2$.

Proof. For each object $(x, K) \in \mathfrak{B}^{\prime}$, let $\rho \downarrow(x, K)$ be the comma category of objects over $(x, K)$. An object in this category is a triple $\left(\left(z^{\prime}, K^{\prime}\right), g\right)$ where $\left(z^{\prime}, K^{\prime}\right) \in \mathfrak{B}^{\prime \prime}$ and $g: \rho\left(z^{\prime}, K^{\prime}\right) \rightarrow(x, K)$ is a morphism in $\mathfrak{B}^{\prime}$. By definition $\rho_{!} \pi_{n}^{F}(\tilde{Y}, \bar{X})(x, K)$ is the colimit over $\rho \downarrow(x, K)$ of the functor that sends $\left(\left(z^{\prime}, K^{\prime}\right), g\right)$ to $\pi_{n}\left(\tilde{Y}_{K^{\prime}}, \bar{X}_{K^{\prime}}, z^{\prime}\right)$. For $K^{\prime}=(x, K) \in \mathfrak{B}^{\prime}$, the objects $\left(\left(z^{\prime}, K^{\prime}\right), 1\right)$ with $z^{\prime} \in \bar{X}_{K^{\prime}}$ are cofinal in the category $\rho \downarrow(x, K)$. Since 
there is a morphism $\left(\left(z_{1}^{\prime}, K^{\prime}\right), 1\right) \rightarrow\left(\left(z_{2}^{\prime}, K^{\prime}\right), 1\right)$ if and only if $z_{1}^{\prime}$ and $z_{2}^{\prime}$ are in the same component of $\bar{X}_{K^{\prime}}$ and these morphisms are isomorphisms, $\rho_{!} \pi_{n}^{F}\left(f^{!} \tilde{Y}, \bar{X}\right)(x, K)=\sum \pi_{i}\left(\widetilde{Y}_{K^{\prime}}, \bar{X}_{K^{\prime}}, z\right)$ where the sum runs over a set $\{z\}$ of representatives for the components of $\bar{X}_{K^{\prime}}$. If we assume the preferred basepoint $\tilde{y}$ of $\widetilde{Y}_{K^{\prime}}$ is in the set, then there is also an inclusion $l: \pi_{n}\left(\widetilde{Y}_{K^{\prime}}, \bar{X}_{K^{\prime}}, \tilde{y}\right) \rightarrow$ $\rho_{!} \pi_{n}^{F}(f ! \tilde{Y}, \bar{X})(x, K)$. By (a), (b), and the analogue of Lemma 1.10 for fragmented spaces, there are integers $M<N$ such that the map $\widetilde{Y}_{K^{\prime}} \rightarrow \widetilde{Y}_{C^{M} K^{\prime}}$ carries all the components of $\bar{X}_{K^{\prime}}$ to the same component of $\bar{X}_{C^{M} K^{\prime}}$ and the map $\widetilde{Y}_{C^{M} K^{\prime}} \rightarrow \widetilde{Y}_{C^{N} K^{\prime}}$ carries any two paths in $\bar{X}_{C^{M} K^{\prime}}$ with the same endpoints to homotopic paths in $\bar{X}_{C^{N} K^{\prime}}$. Hence there is a well-defined homomorphism

$$
\sigma: \sum \pi_{n}\left(\tilde{Y}_{K^{\prime}}, \bar{X}_{K^{\prime}}, z\right) \rightarrow \pi_{n}\left(\tilde{Y}_{C^{N} K^{\prime}}, \bar{X}_{C^{N} K^{\prime}}, \tilde{y}\right) .
$$

Finally, since $p_{(x, K)}:(\tilde{Y}(x, K), \tilde{y}) \rightarrow\left(Y_{K}, x\right)$ is a covering map, there is an isomorphism

$$
p_{*}: \pi_{n}\left(\tilde{Y}_{C^{N} K^{\prime}}, \bar{X}_{C^{N} K^{\prime}}, \tilde{y}\right) \rightarrow \pi_{n}\left(Y_{C^{N} K^{\prime}}, X_{C^{N} K^{\prime}}, x\right)=\pi_{n}^{F}(Y, X)\left[C^{N}\left(x, K^{\prime}\right)\right] .
$$

Then the composite $p_{*} \sigma: \rho_{!} \pi_{n}^{F}\left(f^{!} \tilde{Y}, \bar{X}\right) \rightarrow \pi_{n}^{F}(Y, X)$ is an isomorphism with inverse $l p_{*}^{-1}$.

Lemma 2.2. Let $(Y, X)$ be as in Lemma 2.1. Then the following statements are equivalent:

(a) $f_{*}: \pi_{n}^{F}(X) \rightarrow f^{!} \pi_{n}^{F}(Y)$ is an isomorphism for all $n \geq 0$.

(b) $\tilde{f}_{*}: H_{n}^{F}(\widetilde{X}) \rightarrow f^{!} H_{n}^{F}(\widetilde{Y})$ is an isomorphism for all $n \geq 0$.

Proof. By Proposition 1.5, most of the terms of the exact sequence (1.11)

$\cdots \longrightarrow \pi_{n+1}^{F}(Y, X) \stackrel{\partial}{\longrightarrow} \pi_{n}^{F}(X) \stackrel{f_{*}}{\longrightarrow} f^{!} \pi_{n}^{F}(Y) \stackrel{j_{*}}{\longrightarrow} \pi_{n}^{F}(Y, X) \stackrel{\partial}{\longrightarrow} \pi_{n-1}^{F}(X) \longrightarrow \cdots$

lie in an abelian category. Hence (a) holds if and only if $\pi_{n}^{F}(Y, X)=0$ for all $n \geq 0$. (The special arguments needed to show this for $n \leq 2$ are given in Lemma 4.7 [p. 25].) Since $\widetilde{Y}$ is simply connected, it follows from Lemma $2.1(b)$ that $\bar{X}$ is simply connected. Hence $\rho_{!}: \mathfrak{A b}^{\mathfrak{B} G(\bar{X})}\left(\Sigma^{-1}\right) \rightarrow \mathfrak{A b}^{\mathfrak{B}^{\prime}}\left(\Sigma^{-1}\right)$ is an equivalence of categories by Theorem 5.1 [p. 69], and by Lemma 2.1, $\pi_{n}^{F}(Y, X)=0$ if and only if $\pi_{n}^{F}\left(f^{!} \tilde{Y}, \bar{X}\right)=0$. Since $\widetilde{Y}$ and $\bar{X}$ are simply connected, the Relative Hurewicz Theorem for fragmented complexes, Theorem 8.2 [p. 86], implies that the latter condition holds if and only if $H_{n}^{F}\left(f^{!} \widetilde{Y}, \bar{X}\right)=0$ for all $n \geq 0$. Since the exact sequence of (1.8)

$$
\begin{aligned}
\cdots & \longrightarrow H_{n+1}^{F}\left(f^{!} \tilde{Y}, \bar{X}\right) \stackrel{\partial}{\longrightarrow} H_{n}^{F}(\bar{X}) \stackrel{i_{*}}{\longrightarrow} H_{n}^{F}\left(f^{\prime} \tilde{Y}\right) \\
& \stackrel{j_{*}}{\longrightarrow} H_{n}^{F}\left(f^{\prime} \tilde{Y}, \bar{X}\right) \stackrel{\partial}{\longrightarrow} H_{n-1}^{F}(\bar{X}) \longrightarrow \cdots
\end{aligned}
$$

lies in an abelian category by Proposition $1.5, H_{n}^{F}\left(f^{!} \tilde{Y}, \bar{X}\right)=0$ for all $n \geq 0$ if and only if $\tilde{i}_{*}: H_{n}^{F}(\bar{X}) \rightarrow H_{n}^{F}\left(f^{!} \tilde{Y}\right)$ is an isomorphism for all $n \geq 0$. Let $p^{\prime}(x, K): \widetilde{X}(x, K) \rightarrow X_{K}$ be the universal cover of the component of $X_{K}$ containing $x$ as in Example 1.12. Then for each $(x, K) \in \mathfrak{B}^{\prime}$, there is a map $\tilde{q}(x, K): \widetilde{X}(x, K) \rightarrow \bar{X}(x, K)$ with $p(x, K) \tilde{q}(x, K)=p^{\prime}(x, K)$. Let 
$\tilde{q}: \tilde{X} \rightarrow \bar{X}$ be represented by the family of maps $\left\{q(x, K) \mid(x, K) \in \mathfrak{B}^{\prime}\right\}$. Then $\tilde{\imath} \tilde{q}=\tilde{f}$ covers $f$, and the diagram

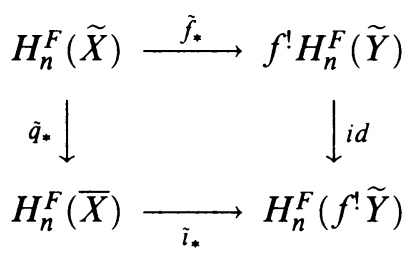

commutes. Since $\bar{X}$ is simply connected, $\tilde{q}$ is an isomorphism of fragmented spaces. Hence $\tilde{q}_{*}$ is an isomorphism. Thus $\tilde{l}_{*}$ is an isomorphism for all $n$ if and only if (b) holds and Lemma 2.2 follows.

Proof of the Boundedly Controlled Whitehead Theorem. By a mapping cylinder argument (cf. the proof of Corollary 10.4 [pp. 96-97]), we may assume $f$ is an inclusion. Since the Whitehead Theorem given in Corollary 10.4 [p. 93] shows that $f:(X, p) \rightarrow(Y, q)$ is a homotopy equivalence if and only if $(Y, q)$ is coextensive with $(X, p)$ and $f_{*}: \pi_{n}^{c}(X, p) \rightarrow f^{!} \pi_{n}^{c}(Y, q)$ is an isomorphism for all $n \geq 0$, it suffices to show that (1)-(3) are equivalent with these conditions. This follows immediately from Lemma 2.2. The proof is complete.

\section{ACKNOWLEDGMENT}

This paper is a revised edition of the original manuscript. The authors would like to thank the referee for suggesting that the paper be made more self-contained and for insisting that it be made readable. His suggestions also resulted in the paper [AM2] in which we prove a bounded and a thin version of the Whitehead Theorem. The first named author would also like to thank the Mathematics Department of the University of Notre Dame for its hospitality during the period when this paper was revised.

\section{REFERENCES}

[AM1] D. R. Anderson and H. J. Munkholm, Boundedly controlled topology, Lecture Notes in Math., vol. 1323, Springer-Verlag, New York and Heidelberg, 1988.

[AM2] _ The Bounded and Thin Whitehead Theorems, Proc. Amer. Math. Soc. 117 (1992), 551-560.

[V] W. Vogell, Boundedly controlled algebraic $K$-theory of spaces and a non-connective delooping of $A(X)$, Universität Bielefeld, preprint, 1989.

Department of Mathematics, Syracuse University, Syracuse, New York 13244

Institut for Matematik og Datalogi, Odense Universitet, DK 5230 Odense M, DenMARK 